\title{
CD90 promotes cell migration, viability and sphere-forming ability of hepatocellular carcinoma cells
}

\author{
KETAO ZHANG ${ }^{1,2^{*}}$, SIYAO $\mathrm{CHE}^{3^{*}}$, ZHENG SU$^{4}$, SHANGYOU ZHENG $^{2}$, \\ HUAYAO ZHANG $^{5}$, SHANGLIN YANG ${ }^{6}$, WENDA LI $^{7}$ and JIANPING LIU ${ }^{1,2}$ \\ ${ }^{1}$ Guangdong Provincial Key Laboratory of Malignant Tumor Epigenetics and Gene Regulation; \\ ${ }^{2}$ Department of Hepatopancreatobiliary Surgery, Sun Yat-Sen Memorial Hospital of Sun Yat-Sen University, Guangzhou, \\ Guangdong 510120; ${ }^{3}$ Department of Hepatobiliary Surgery, Gaozhou People's Hospital, Gaozhou, Guangdong 525200; \\ ${ }^{4}$ Department of Hepatobiliary Surgery, Guizhou Provincial People's Hospital, Guiyang, Guizhou 550000; \\ ${ }^{5}$ Department of Mammary Gland and Thyroid Gland Surgery, The Third People's Hospital of Dongguan, Dongguan, \\ Guangdong 523000; ${ }^{6}$ Department of Hepatobiliary Surgery, Affiliated Foshan Hospital of Southern Medical University, \\ Foshan, Guangdong 528000; ${ }^{7}$ Department of Hepatobiliary Surgery, \\ Sun Yat-Sen Memorial Hospital of Sun Yat-Sen University, Guangzhou, Guangdong 510120, P.R. China
}

Received July 17, 2017; Accepted November 29, 2017

DOI: $10.3892 / \mathrm{ijmm} .2017 .3314$

\begin{abstract}
Cluster of differentiation (CD)90 (Thy-1) was proposed as a marker for the liver cancer stem cells that are responsible for tumorigenic activity, however its involvement in the progression of hepatocellular carcinoma (HCC) remains unknown. The aim of the present study was to determine the effect of CD90 on the biological functions of HCC and to investigate the associated circular RNA (circRNA) involved in this process. The analysis of the in vitro data demonstrated that $\mathrm{CD} 0^{+}$cells isolated from SK-Hep-1 cells exhibited increased viability, migration and invasive abilities compared with $\mathrm{CD}^{-} 0^{-}$cells. In addition, circRNA expression profiles in $\mathrm{CD}^{+}{ }^{+}$and CD90- cells were screened using a microarray assay and hsa_circ_0067531 and hsa_circ_0057096 were identified to be expressed at significantly different levels. It was additionally demonstrated that the expression of hsa_circ_0067531 in HCC tissues was significantly decreased compared with normal adjacent tissues. Overall, the results of the present study suggested that CD90 may be used as a potential biomarker for HCC. Furthermore, it was demonstrated that hsa_circ_0067531 may affect the biological functions of
\end{abstract}

Correspondence to: Dr Jianping Liu, Guangdong Provincial Key Laboratory of Malignant Tumor Epigenetics and Gene Regulation, Sun Yat-Sen Memorial Hospital of Sun Yat-Sen University, 107 Yanjiang Road, Guangzhou, Guangdong 510120, P.R. China E-mail: liuzhunlong@126.com

*Contributed equally

Key words: hepatocellular carcinoma, cluster of differentiation 90, circular RNA
$\mathrm{CD} 90^{+} \mathrm{HCC}$ cells and may be a promising candidate to aid in the diagnosis and therapy of HCC.

\section{Introduction}

Hepatocellular carcinoma (HCC) ranks as the fifth most frequently occurring neoplasm and the second leading cause of cancer-associated mortalities worldwide. A total of $>745,000$ fatalities occur as a result of HCC, along with 500,000 newly diagnosed cases every year (1-3). Current diagnostic standards may be inaccurate and result in missed cases, and since early diagnosis is key for successful operations, it is imperative to search for more efficient biomarkers to increase the early diagnostic rate of $\mathrm{HCC}(4,5)$. Cancer stem cells (CSCs) are the source of numerous solid tumor types, including $\mathrm{HCC}$, and are characterized by a variety of stem cell markers (6-11). A previous study revealed that cluster of differentiation (CD)90 (Thy-1) is a potential marker for liver CSCs (12) and is expressed in a variety of cells, including T-cells, thymocytes, neurons, endothelial cells and fibroblasts. It is important in cell-to-cell and cell-to-matrix interactions, apoptosis, adhesion, migration and fibrosis (13). Previous studies have demonstrated that $\mathrm{CD}^{+} 0^{+}$, however not CD90cells, obtained from HCC cell lines, exhibit tumorigenic and metastatic capacities $(12,14,15)$. The human HCC cell line JHH-6 demonstrates increased proliferative capacity of CD90 ${ }^{+}$ compared with CD90- cells (16). Conversely, CD90 functions as a tumor suppressor in ovarian cancer (17). The specific function and mechanism by which $\mathrm{CD} 90^{+}$cells contribute to HCC progression remains to be elucidated.

Circular RNAs (circRNAs) are a novel type of endogenous noncoding RNA characterized as stable, abundant, and conserved, and usually exhibit tissue/developmental-stage specific expression (18). Numerous studies have revealed that circRNAs participate in the initiation and development of multiple diseases, including Alzheimer's, Parkinson's, 
atherosclerosis, and various cancers $(19,20)$. CircRNAs bind to miRNAs, acting as miRNA sponges, and thereby regulate gene expression (21). Hsa_circ_0004018 has been reported to be involved in cancer-associated pathways in HCC via interactions with miRNAs (22). The circRNA that acts as a sponge for miR-7 is Cdrlas (ciRS-7), the knockdown of which suppresses HCC cell viability and invasion (23). This evidence suggests that circRNAs are involved in HCC progression and may be promising diagnostic or predictive biomarkers. However, the expression of circRNAs in HCC has not extensively been studied and the mechanisms of circRNAs in HCC remain to be elucidated.

The present study aimed to investigate the effect of CD90 on the cell cycle and the migration, invasion, and sphere formation abilities of HCC cells. Furthermore, differentially expressed circRNAs between $\mathrm{CD}^{+} 0^{+}$and $\mathrm{CD}^{-} 0^{-} \mathrm{HCC}$ cells were identified via high throughput microarray assay.

\section{Materials and methods}

Ethics statement. The present study was approved by the Ethics Committee of Sun Yat-Sen University (Guangzhou, China), and written informed consent was obtained from all patients in Sun Yat-Sen Memorial Hospital of Sun Yat-Sen University.

Specimen collection and cell culture. A total of eight pairs of HCC tumor tissues and corresponding non-tumor tissues were collected from the Department of Hepatopancreatobiliary Surgery, Sun Yat-Sen Memorial Hospital of Sun Yat-Sen University. The present study included 8 patients with HCC, 5 males and 3 females, aged 35-66 years with a mean age of $55.9 \pm 9.5$ years. Exclusion criteria were the following: i) Patients with moderate or tense ascites, ii) patients refusing liver biopsy, iii) patients with previous treatment for HCC and iv) patients with a history of severe trauma. None of the recruited participants accepted adjunctive treatment prior to the surgery. All tissue samples were immediately preserved at $-80^{\circ} \mathrm{C}$ following washing with sterile phosphate-buffered saline (PBS). HCC cell lines (LM3, Huh7, MHCC 97L and SK-Hep-1) were purchased from Shanghai Cell Center (Shanghai, China) and cultured in Dulbecco's modified Eagle's medium (DMEM; Invitrogen; Thermo Fisher Scientific, Inc., Waltham, MA, USA) supplemented with $10 \%$ fetal calf serum (Sigma-Aldrich; Merck KGaA, Darmstadt, Germany) and $1 \%$ penicillin-streptomycin $\mathrm{G}$ (Invitrogen; Thermo Fisher Scientific, Inc.). Cells were incubated at $37^{\circ} \mathrm{C}$ in a humidified atmosphere containing $5 \% \mathrm{CO}_{2}$.

Flow cytometry. The quantitation of CD90 expression in the cells (Huh7, MHCC 97L and SK-Hep-1) was determined using flow cytometry. A total of $2 \times 10^{5}$ cells were transferred into a centrifuge tube and suspended in PBS buffer. Following centrifugation at $1,000 \times \mathrm{g}$ for $5 \mathrm{~min}$ at $4^{\circ} \mathrm{C}$, the cells were fixed with fresh fixative (4\% paraformaldehyde; Thermo Fisher Scientific Inc.) at room temperature for $15 \mathrm{~min}$, and then preincubated in $200 \mathrm{ml}$ blocking solution (10\% goat serum containing 0.05\% Tween-20; Thermo Fisher Scientific Inc.) for $1 \mathrm{~h}$ at room temperature. The cells were incubated with fluorescein isothiocyanate (FITC)-conjugated human anti-CD90 (catalog no. 85-11-0909-41; 1:100 dilution;
eBiosciences; Thermo Fisher Scientific Inc.) for $1 \mathrm{~h}$ at $4^{\circ} \mathrm{C}$, then the cells were washed in PBS and incubated with FITC-conjugated goat anti-mouse IgG secondary antibody (catalog no. ab6785; 1:1,000 dilution; Abcam, Cambridge, MA, USA). The standard was established by isotype match control (FITC-conjugated mouse IgG1 isotype control, catalog no. 85-11-4714-81; 1:100 dilution; eBiosciences; Thermo Fisher Scientific Inc.) for $1 \mathrm{~h}$ at $4^{\circ} \mathrm{C}$. A total of $1 \times 10^{6}$ FITC-labeled cells were measured using BD AccuriC6 Flow Cytometer with Cell Quest software version 5.1 (BD Biosciences, Franklin Lakes, NJ, USA). Each experiment was performed in triplicate. The cell cycle of SK-Hep-1 CD90 ${ }^{+}$and CD90- cells was additionally determined with flow cytometry. Briefly, cells were resuspended in PBS twice prior to fixation by dropwise addition into $95 \%$ pre-cooled ethanol for $15 \mathrm{~min}$ at room temperature, and then centrifuged at 3,000 x g for $10 \mathrm{~min}$ at $4^{\circ} \mathrm{C}$, resuspended twice in PBS, and stained with propidium iodide (PI) containing $50 \mu \mathrm{g} / \mathrm{ml}$ RNaseA (Takara Bio, Inc., Otsu, Japan) in the dark for $30 \mathrm{~min}$ at room temperature. The DNA content was analyzed using BD AccuriC6 Flow Cytometer with Cell Quest software, version 5.1 (BD Biosciences).

Cell separation via magnetic sorting. Separation of $\mathrm{CD} 90^{+}$ cells from SK-Hep-1 cells was performed using MACS magnetic cell sorting (Miltenyi Biotech $\mathrm{GmbH}$, Bergisch Gladbach, Germany) according to the manufacturer's protocol. SK-Hep-1 cells were incubated with $50 \mu \mathrm{l}$ of FcR blocking agent (Miltenyi Biotech $\mathrm{GmbH}$ ) and CD90-biotin immunomagnetic beads (Miltenyi Biotech $\mathrm{GmbH}$ ) for $10 \mathrm{~min}$ at $4^{\circ} \mathrm{C}$. Goat anti-mouse $\mathrm{IgG}$ magnetic beads (Miltenyi Biotech $\mathrm{GmbH}$ ) were used as the control. A MACS cell separation column (MS column) was used to retain the CD90 ${ }^{+}$cells linked to the beads. Unlabeled cells (SK-Hep-1 CD90- cells) were washed with $3 \times 500 \mu$ l buffer (containing PBS, $0.5 \%$ BSA, 2 mM EDTA) and collected. The MS column was then removed from the separator and placed on another collection tube. A total of $1 \mathrm{ml}$ buffer was pipetted onto the MS column, and the labeled CD90 ${ }^{+}$cells (SK-Hep-1 CD90 ${ }^{+}$ cells) were flushed out by applying the plunger supplied with the column. The labeled CD90 ${ }^{+}$cells were eventually obtained via centrifugation at $3,000 \mathrm{x}$ g for $10 \mathrm{~min}$ at $4^{\circ} \mathrm{C}$ and resuspension in serum-free medium (Gibco; Thermo Fisher Scientific, Inc.). The SK-Hep-1 CD90+ and SK-Hep-1 CD90- cells were harvested and washed with PBS, and the expression of CD90 in these cells was directly investigated with flow cytometry. Following sorting, the SK-Hep- $1 \mathrm{CD}^{\circ} 0^{+}$cells indicated an $\geq 85 \%$ expression level of CD90 and the SK-Hep-1 CD90- cells revealed $<5 \%$ expression level of CD90.

Western blotting. SK-Hep-1 cells were lysed in lysis buffer (Gibco; Thermo Fisher Scientific, Inc.) and the concentration of total protein was analyzed using a BCA Protein Assay Kit (Sangon Biotech Co., Ltd, Shanghai, China). A total of $30 \mu \mathrm{g}$ protein was added into each lane of the $10 \%$ SDS-PAGE gel and 5\% skim milk (BD Biosciences) was used to block the polyvinylidene membranes for $10 \mathrm{~min}$ at room temperature. The membranes were then incubated with the primary antibody anti-CD90/Thy1 (catalog no. ab133350; 1:100; Abcam) at $4^{\circ} \mathrm{C}$ overnight. GAPDH was used as the internal 
control (anti-GAPDH antibody; catalog no. ab9485; 1:100, Abcam). The next day, the membranes were incubated with horseradish peroxidase-conjugated anti-rabbit $\operatorname{IgG}$ secondary antibody for $1 \mathrm{~h}$ at room temperature (1:500 dilution; catalog no. ab7090; Abcam). The results were visualized using the enhanced chemiluminescence substrate kit (GE Healthcare, Chicago, IL, USA). All images were analyzed with ImageJ software, version 14.8 (National Institutes of Health, Bethesda, MD, USA).

Cell viability. Cell viability was assessed with a 3-(4,5-dim ethylthiazol-2-yl)-2,5-diphenyl-trtrazolium bromide (MTT) assay. The treated cells were seeded into a 96 -well plate and cultured in complete medium. When the cells adhered, $0.5 \mathrm{mg} / \mathrm{ml}$ MTT was added to each well and incubated at $37^{\circ} \mathrm{C}$ for $4 \mathrm{~h}$. The supernatant was then carefully aspirated and $100 \mu \mathrm{l}$ of dimethyl sulfoxide was added. The absorbance was measured at a wavelength of $490 \mathrm{~nm}$ using a microplate reader (Thermo Fisher Scientific, Inc.).

Migration and invasion assays. Cell migration and invasion assays were performed using Transwell chambers with $8 \mu \mathrm{m}$ pores (BD Biosciences) according to the manufacturer's protocol. For migration assays, SK-Hep-1 CD90 ${ }^{+}$ $\left(5 \times 10^{4}\right.$ cells/well) and SK-Hep-1 CD90- cells $\left(5 \times 10^{4}\right.$ cells/well) were separately seeded into the upper compartment without Matrigel, for invasion assay, the cells were seeded into the upper compartment with Matrigel (Corning Inc., Corning, NY, USA) and incubated in serum-free DMEM (Gibco; Thermo Fisher Scientific, Inc.) and the lower compartment was filled with complete medium supplemented with $10 \%$ FBS. Following $24 \mathrm{~h}$, migratory and invasive cells on the bottom surface of the filters were fixed with $4 \%$ paraformaldehyde for $24 \mathrm{~h}$ and stained with $0.1 \%$ crystal violet solution for $10 \mathrm{~min}$ at room temperature. The cells in at least 10 randomly selected microscopic fields were counted under a light microscope, at x200 magnification (Nikon Corp., Tokyo, Japan). Each experiment was performed in triplicate.

Sphere formation assay. SK-Hep-1 CD90 ${ }^{+}$and SK-Hep-1 CD90- cells were trypsinized and cultured in DMEM-F12 (Sigma-Aldrich; Merck KGaA) that was supplemented with $20 \mathrm{ng} / \mathrm{ml}$ EGF, $10 \mathrm{ng} / \mathrm{ml}$ FGF, $4 \mathrm{ng} / \mathrm{ml}$ insulin, and B27 (1:50) in Ultra-Low Attachment 6-well plates (Corning Inc.). The cells were imaged under a light microscope (magnification, x200; Olympus Corp., Tokyo, Japan) following 20 days of incubation.

Microarray hybridization. Total RNA was treated with Rnase R (Epicentre Illumina, Inc., San Diego, CA, USA) to remove linear RNA, and was then amplified and transcribed into fluorescent cRNA using Quick Amp Labeling kit (Takara Bio, Inc.) according to the manufacturer's protocol. The cRNA was then purified with the RNeasy Mini Kit (Qiagen GmbH, Hilden, Germany) and hybridized to the circRNA expression microarray according to the manufacturer's protocols. The microarray hybridization and the data collection were performed by Forevergen Bio-tech Co. (Guangzhou, China). For the microarray analysis, following filtering by flag signal, the raw data were processed and normalized.
Differentially expressed circRNA were identified with $\mathrm{P}<0.05$ and fold changes $>2$. In addition, Kyoto Encyclopedia of Genes and Genomes (KEGG) pathway analyses was performed which provided more detailed information regarding the pathways in which the target genes were involved.

Specialty confirmation and Sanger sequencing. To verify the specialty of the PCR products of hsa_circ_0067531 and hsa_circ_0057096, the cDNA and gDNA PCR products that had been amplified by diverted primers were separated on a $2 \%$ agarose gel with TE running buffer. Only a single band in the gel was thought to be a specialty. The region was incised from the whole gel and purified with SanPrep Column DNA Gel Extraction Kit (Qiagen GmbH), and the PCR fragments were inserted into $\mathrm{T}$ vector for Sanger sequencing (Tsingke Biotech Co., Ltd., Beijing, China).

Quantitative polymerase chain reaction ( $q P C R)$. Total RNA was isolated using TRIzol ${ }^{\circledast}$ reagent (Invitrogen; Thermo Fisher Scientific, Inc.) following indicated treatments. qPCR assays were performed with SYBR Premix Ex Taq (Takara Bio, Inc.), primers, and a cDNA template on the Applied Biosystems 7500 Real-time PCR System (Applied Biosystems; Thermo Fisher Scientific, Inc.). The primers used in the assay are listed as follows: Forward, CGAACCAACTTCACCAGCAA and reverse, CTGATGCCCTCACACTTGAC for CD90; forward, 5'-TCCAGACCAGTACGTTCGAG-3' and reverse, 5'-TCA CACAGTTGAGACAAGGGAT-3' for cir_0067531; forward, 5'-AGGCAAAGGAAGTCCATCTCAT-3' and reverse, 5'-TCAATCACACCCTGGGCCAT-3' for circ_0057096 and forward, 5'-CCGAGAATGGGAAGCTTGTC-3' and reverse, 5'-AAGCACCAACAGAGGAGAA-3' for GAPDH. PCR was conducted in a $50 \mu \mathrm{l}$ reaction system, including $1 \mu \mathrm{l}$ forward and $1 \mu \mathrm{l}$ reverse primers, $0.5 \mu \mathrm{l}$ 20x SYBR-Green I, $25 \mu \mathrm{l}$ 2xPCR buffer, $2 \mu \mathrm{l}$ cDNA, and $20.5 \mu \mathrm{l}$ DEPC. The qPCR program was $94^{\circ} \mathrm{C}$ for $4 \mathrm{~min}, 30$ cycles of $94^{\circ} \mathrm{C}$ for $20 \mathrm{sec}, 60^{\circ} \mathrm{C}$ for $30 \mathrm{sec}$, $72^{\circ} \mathrm{C}$ for $30 \mathrm{sec}$, and $72^{\circ} \mathrm{C}$ for $5 \mathrm{~min}$. Each individual sample was run in triplicate and the expression level was quantified with the comparative cycle threshold (Cq) method. Results were normalized to GAPDH expression and RNA enrichments were calculated using the $2^{-\Delta \Delta \mathrm{Cq}}$ method (24).

Statistical analysis. Quantile normalization and subsequent data processing were performed using the GeneSpring GX software, version 11.5.1 package (Agilent Technologies, Inc., Santa Clara, CA, USA). Other data were analyzed with the two-tail Student's t-test and one-way analysis of variance followed by Tukey's test. SPSS software, version 19.0 (IBM SPSS, Armonk, NY, USA). Each experiment was repeated at least three times. All results were summarized and are presented as the mean \pm standard deviation. $\mathrm{P}<0.05$ was considered to indicate a statistically significant difference.

\section{Results}

Distribution of CD90 cells in human HCC cell lines. The expression levels of CD90 in the 4 different HCC cell lines were determined by qPCR; results of which demonstrated that the expression of CD90 was increased in the LM3, Huh7, 
A

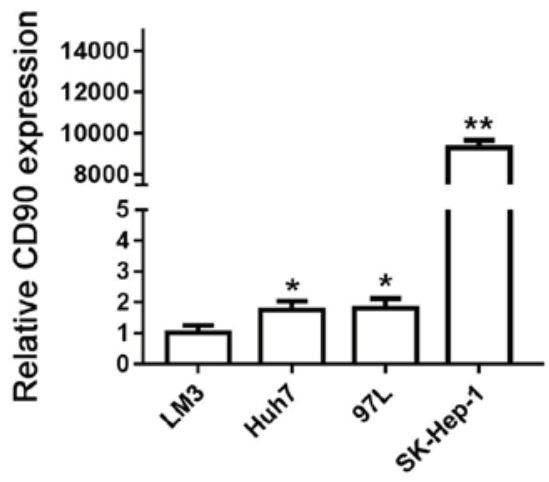

B
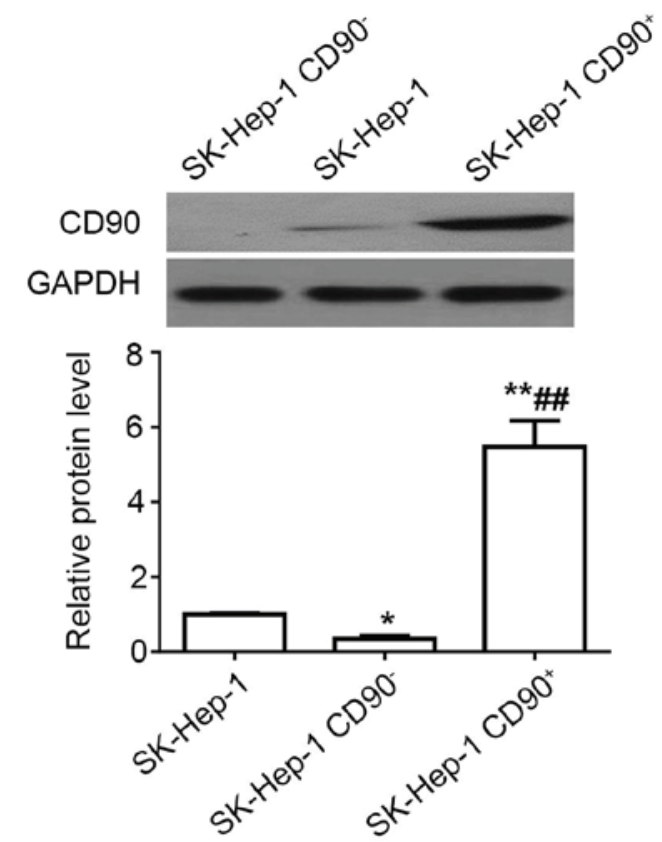

C

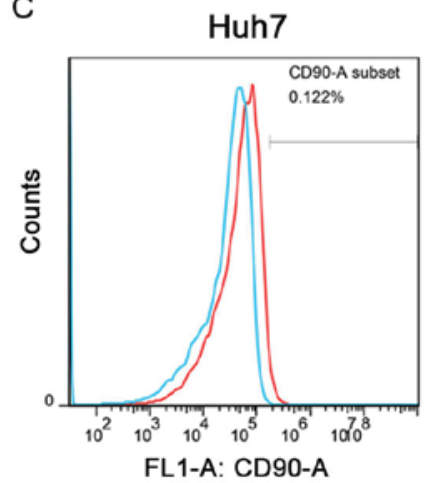

SK-Hep-1 CD90+

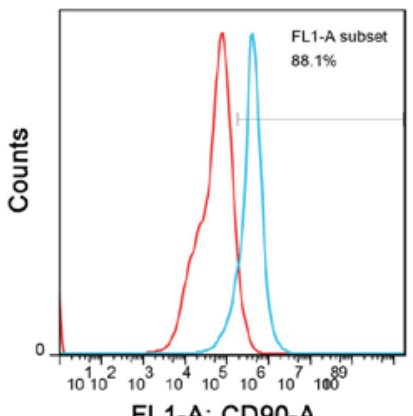

97L

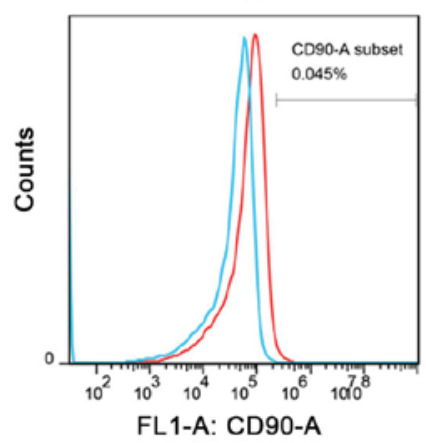

SK-Hep-1 CD90

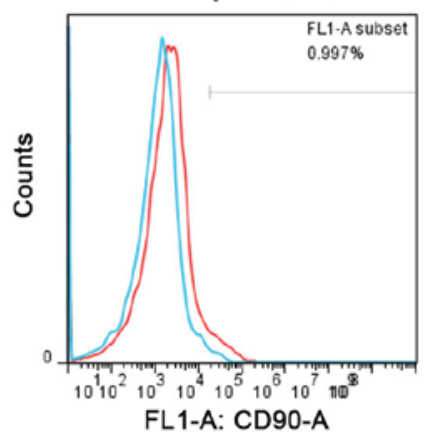

SK-Hep-1
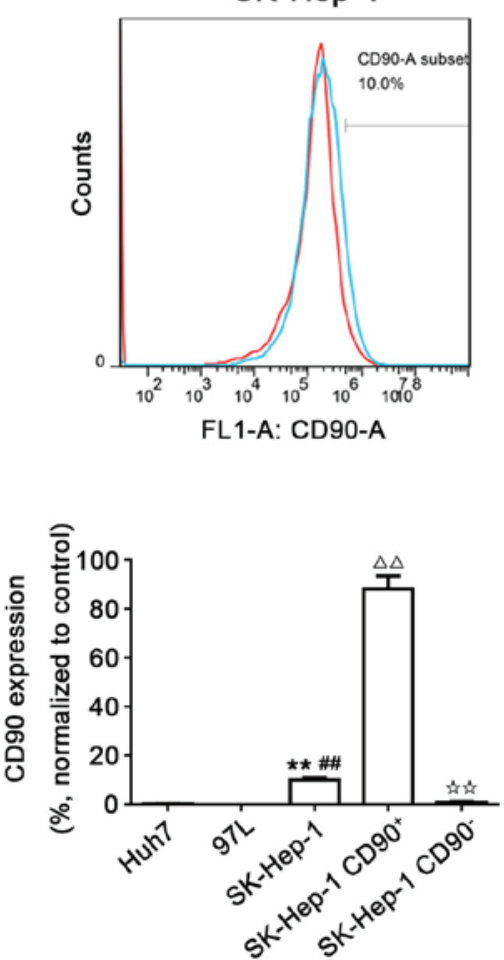

Figure 1. Distribution of CD90 cells in human HCC cell lines. (A) Expression levels of CD90 in 4 different HCC cell lines (LM3, Huh7, MHCC 97L and

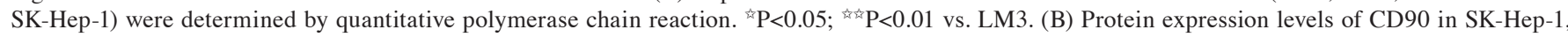
SK-Hep-1 CD90 ${ }^{-}$, and SK-Hep-1 CD90 ${ }^{+}$cells were determined by western blotting. GAPDH was used as the internal control. ${ }^{\text {齐 }} \mathrm{P}<0.05$; ${ }^{\# \#} \mathrm{P}<0.01$ vs. SK-Hep-1 CD90- (C) Expression levels of CD90 in 3 different HCC cell lines (Huh7, MHCC 97L, and SK-Hep-1), SK-Hep-1 CD90 cells and SK-Hep-1 CD90- cells were examined with flow cytometry. Each experiment was performed in triplicate. IgG was used for control (red line). The blue line reveals the expression of CD90. The SK-Hep-1 CD90+ cells revealed an expression of 88.1\% CD90, whereas CD90 cells exhibited a CD90 expression of $0.997 \%$. ${ }^{\text {sin }} \mathrm{P}<0.01$ vs. Huh7; ${ }^{\# \#} \mathrm{P}<0.01$ vs. MHCC $97 \mathrm{~L} ;{ }^{\triangle \Delta} \mathrm{P}<0.01$ vs. SK-Hep-1; ${ }^{\text {th }} \mathrm{P}<0.01$ vs. SK-Hep-1 CD90 ${ }^{+}$. CD, cluster of differentiation; HCC, hepatocellular carcinoma.

and MHCC 97L cell lines, and particularly in the SK-Hep-1 cell line (Fig. 1A). The western blotting results demonstrated that the protein level of CD90 in SK-Hep-1 CD90 ${ }^{+}$ cells was significantly increased compared with SK-Hep-1 and SK-Hep-1 CD90- cells. However, CD90 expression in
SK-Hep-1 CD90- cells was significantly decreased compared with SK-Hep-1 cells (Fig. 1B). In addition, the expression of CD90 (blue line) and control $\operatorname{IgG}$ (red line) were verified in the Huh7, MHCC 97L, and SK-Hep-1 cell lines by flow cytometry. It was demonstrated that the relative expression level of 
A

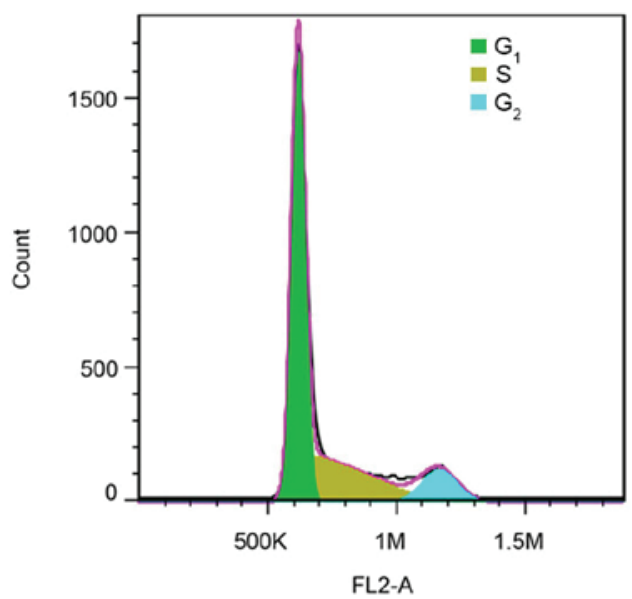

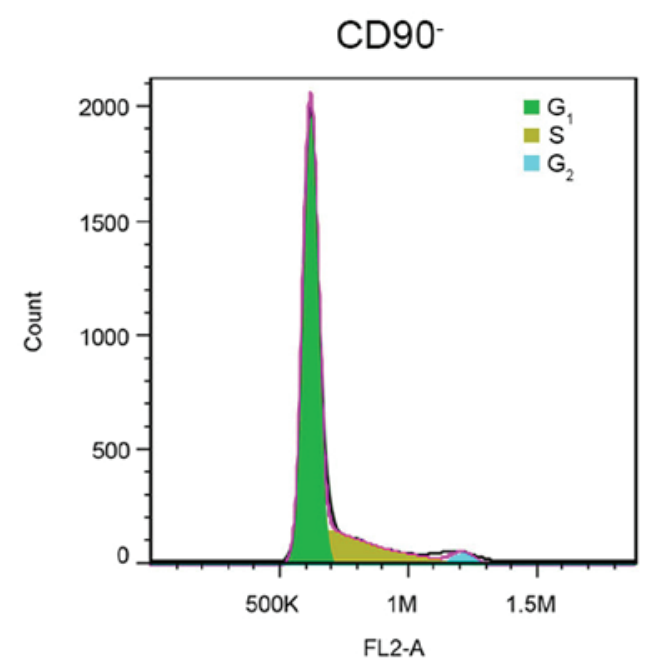

B

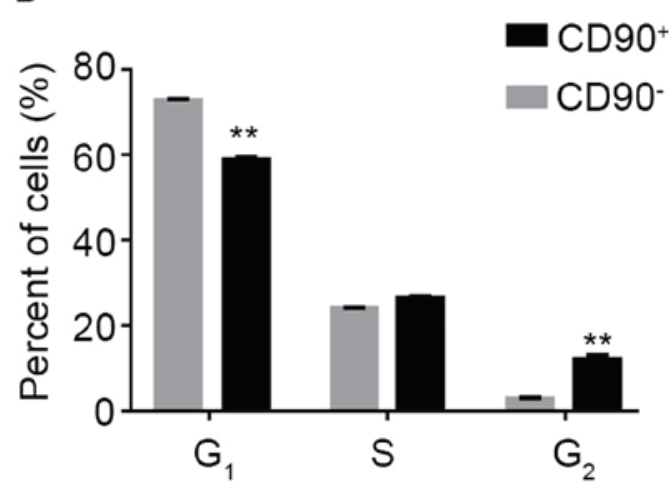

$\mathrm{C}$

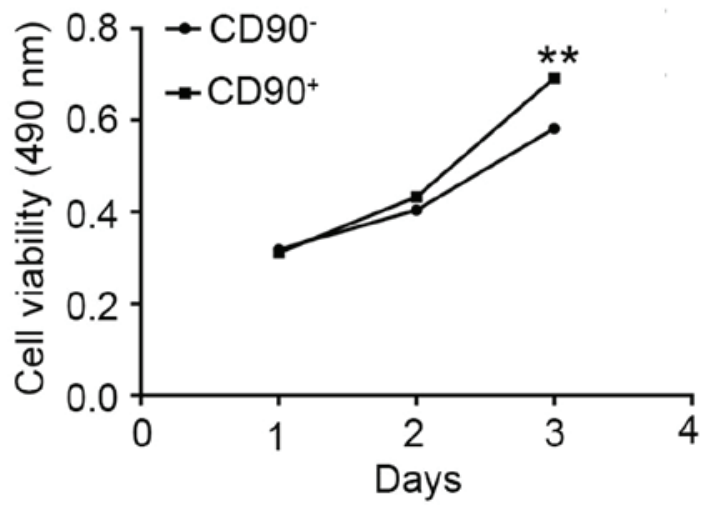

Figure 2. Effect of SK-Hep-1 CD90 ${ }^{+}$cells on cell cycle and viability. (A) Representative image and (B) quantitative analysis of flow cytometry assay to analyze the cell cycle of CD90 ${ }^{+}$and CD90- cells. Data are presented as the mean \pm standard deviation of three independent experiments. (C) MTT assay used to determine the viability of $\mathrm{CD} 90^{+}$and $\mathrm{CD}^{-} 0^{-}$cells. The results demonstrated that the viability of $\mathrm{CD} 90^{+}$cells was significantly increased compared with CD90- cells. ${ }^{* *} \mathrm{P}<0.01$ vs. CD90-. CD, cluster of differentiation.

CD90 in SK-Hep-1 cells (10.0\%) was significantly increased compared with Huh7 (0.122\%) and MHCC 97L (0.045\%) cell lines (Fig. 1C). Consequently, the SK-Hep-1 cell line was selected for the ensuing experiments. The $\mathrm{CD} 90^{+}$cells were sorted from the SK-Hep-1 cell line with an expression of CD90 $(88.1 \%) \geq 85.0 \%$, whereas CD90- cells were sorted from the SK-Hep-1 cell line with an expression of CD90 (0.997\%) $\leq 5.0 \%$ (Fig. 1C).

Effect of CD90 on the cell cycle and viability of SK-Hep-1 cells. Flow cytometry analysis and MTT assay were performed to investigate the effect of CD90 on the cell cycle and viability in SK-Hep-1 cells. The raw FACScan data is presented in Fig. 2A. The numerical conversion of the cell population in $\mathrm{G}_{1}, \mathrm{~S}$, and $\mathrm{G}_{2}$ phases is presented in Fig. 2B. The results indicated that the percentage of $\mathrm{CD} 90^{+}$cells was significantly decreased in the $\mathrm{G}_{1}$ phase, compared with $\mathrm{CD}^{-} 0^{-}$cells. Conversely, the percentage of $\mathrm{CD}^{+} 0^{+}$cells was significantly increased in the $\mathrm{G}_{2}$ phase, compared with $\mathrm{CD} 90^{-}$cells. These results suggested that the $\mathrm{G}_{1}$ phase of $\mathrm{CD} 90^{+}$cells was shorter compared with CD90- cells. In addition, the MTT assay results demonstrated that the cell viability of $\mathrm{CD} 90^{+}$cells was significantly increased compared with CD90- cells at day 3 (Fig. 2C).
Migration and invasion abilities increase in $\mathrm{CD}^{+}$cells. Transwell assays were used to determine the migration and invasion abilities of $\mathrm{CD}^{+}$and $\mathrm{CD}^{-} 0^{-}$cells. The migration assay revealed that there were significantly more $\mathrm{CD}^{+} 0^{+}$cells compared with CD90- cells (Fig. 3A). Similarly, the invasion assay demonstrated the same results as the migration assay (Fig. 3B). Consequently, it was demonstrated that CD90 ${ }^{+}$ cells exhibited increased migration and invasion capabilities compared with CD90 cells.

$C D 90^{+}$induces sphere formation. The sphere formation assay demonstrated that $\mathrm{CD}^{-} 0^{-}$cells did not form spheres following 20 days of incubation, however the CD90+ cells did (Fig. 4).

Identification of differentially expressed circRNA profiles. A high throughput microarray assay was used to identify the differing expressions of circRNAs in CD90 ${ }^{+}$and CD90cells. In the present study, 274 dysregulated circRNAs were identified, including 238 upregulated and 36 downregulated circRNAs (Fig. 5A). The upregulated circRNAs were more frequently occurring compared with downregulated circRNAs. In order to further understand the biological functions of the differentially expressed circRNAs, KEGG pathway analyses 
A

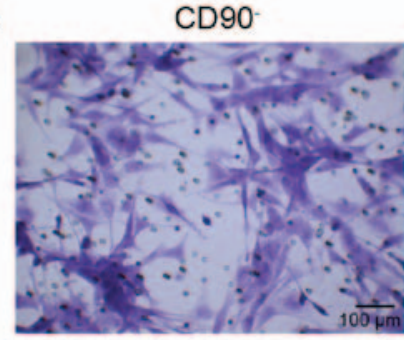

B

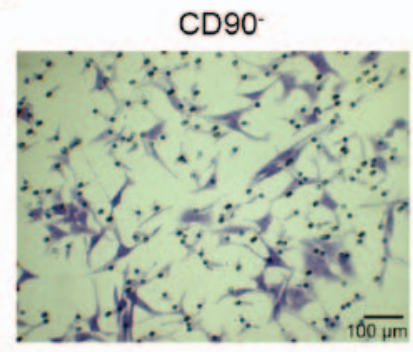

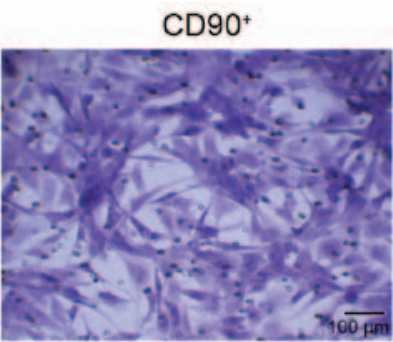
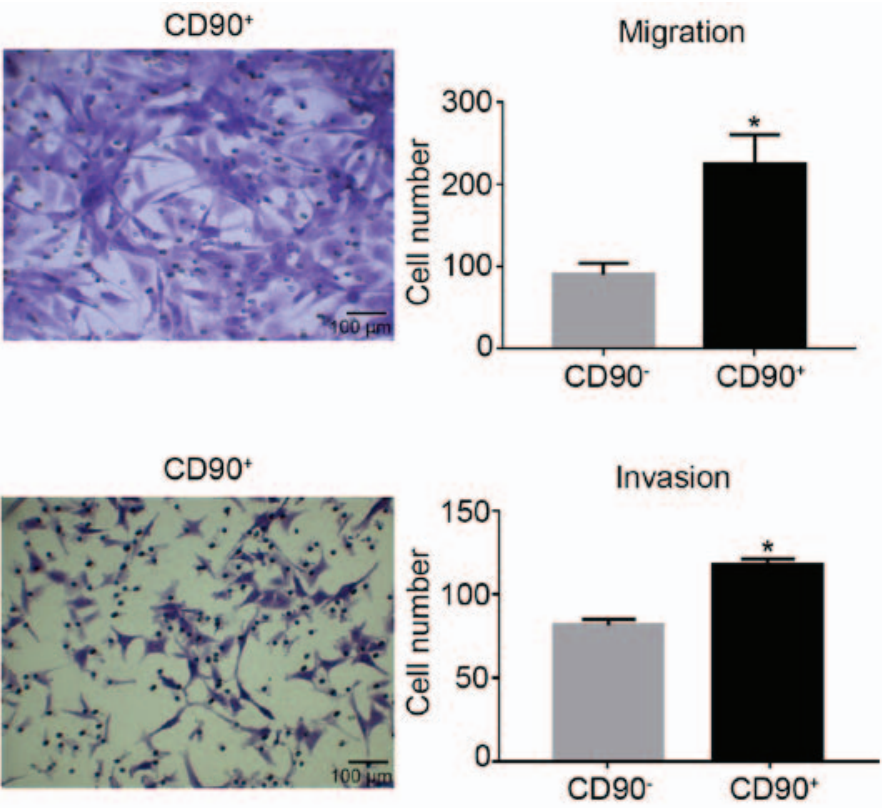

Figure 3. Migration and invasion ability of $\mathrm{CD} 90^{+}$and $\mathrm{CD} 90^{-}$cells. (A) A Transwell assay was used to determine the migration ability of $\mathrm{CD} 90^{+}$and $\mathrm{CD} 90^{-}$cells. (B) A Matrigel Transwell assay was used to determine the invasion ability of $\mathrm{CD} 90^{+}$and $\mathrm{CD} 90^{-}$cells. The results demonstrated that the migration and invasion abilities of $\mathrm{CD} 90^{+}$cells were significantly increased compared with $\mathrm{CD} 90^{-}$cells. " $\mathrm{P}<0.05$ vs. CD90- Magnification, $\mathrm{x} 200$; Scale bar, $100 \mu \mathrm{m}$. CD, cluster of differentiation.
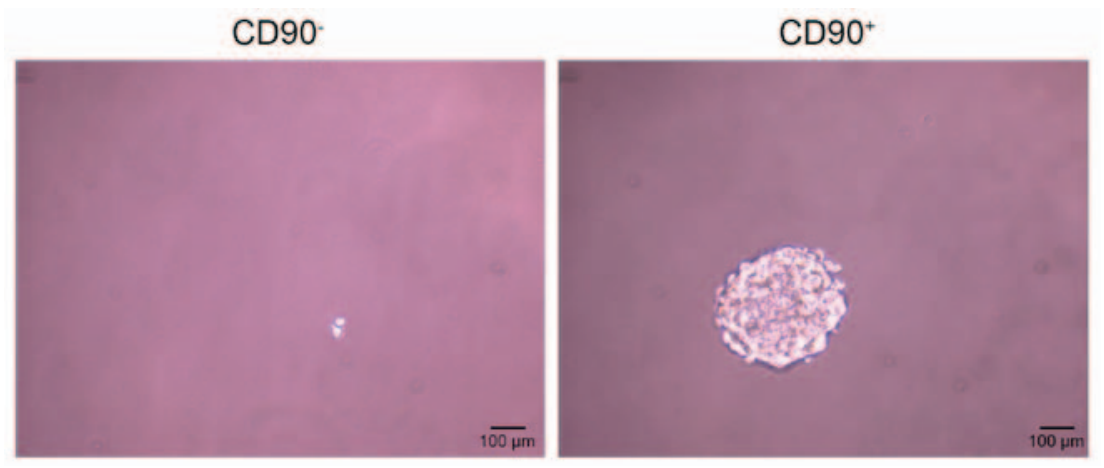

Figure 4. Sphere-forming ability of $\mathrm{CD} 90^{+}$and $\mathrm{CD} 90^{-}$cells. Spheroid formation assay. $\mathrm{CD} 90^{-}$and $\mathrm{CD} 90^{+}$cells were cultured on 6-well plates or as cell spheres. The cells were imaged under a light microscope (magnification, x200) following 20 days of incubation. Each experiment was repeated three times. Scale bar, $100 \mu \mathrm{m}$.

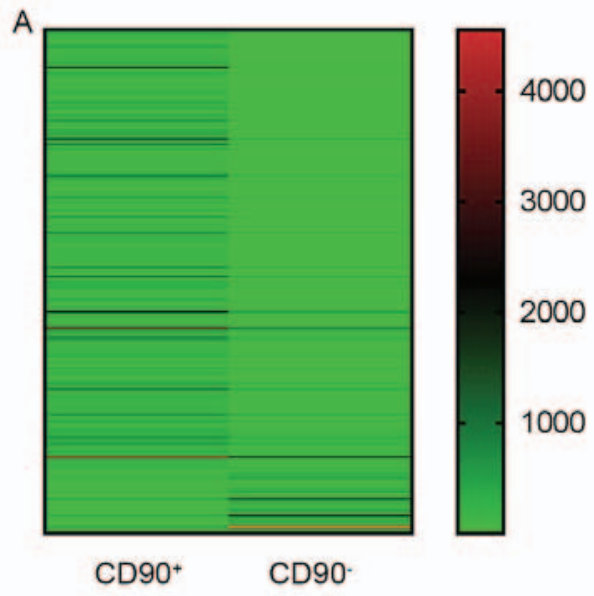

B
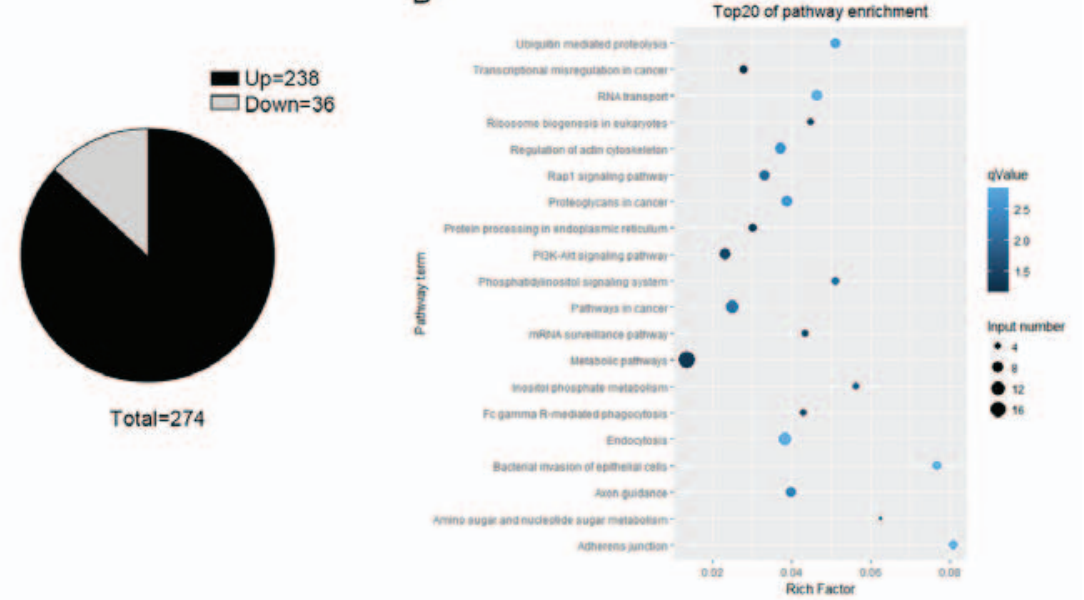

Figure 5. Identification of differentially expressed circRNA profiles. Differentially expressed circRNAs between CD90 ${ }^{+}$and CD90- cells were analyzed using a high throughput microarray assay. (A) The heat map revealed 274 distinguishable (238 upregulated and 36 downregulated) circRNAs with differential expression between $\mathrm{CD} 90^{+}$and CD90- cells. (B) The key signaling pathways for these circRNAs were identified by Kyoto Encyclopedia of Genes and Genomes enrichment analyses and the top 20 are listed; the top three signaling pathways were the metabolic pathway, pathways in cancer, and the P13K-AKT signaling pathway. CD, cluster of differentiation; circRNA, circular RNA; P13K-AKT, phosphoinositide 3-kinase (P13K)-RAC- $\alpha$ serine/threonine-protein kinase. 
A

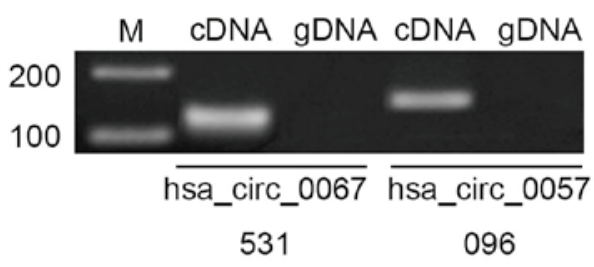

C

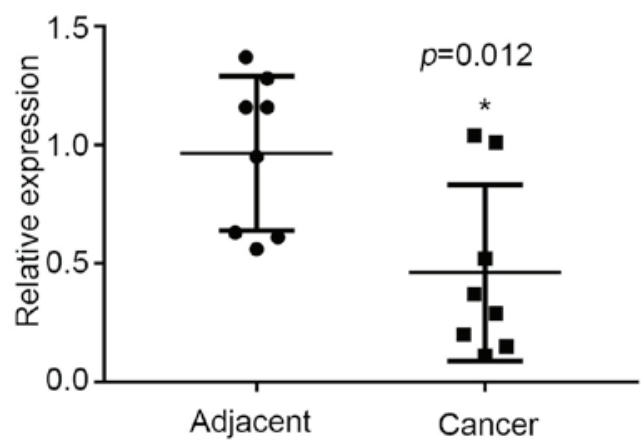

B

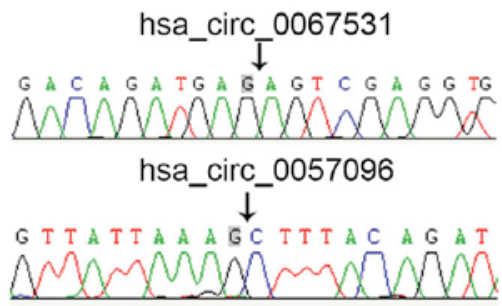

D

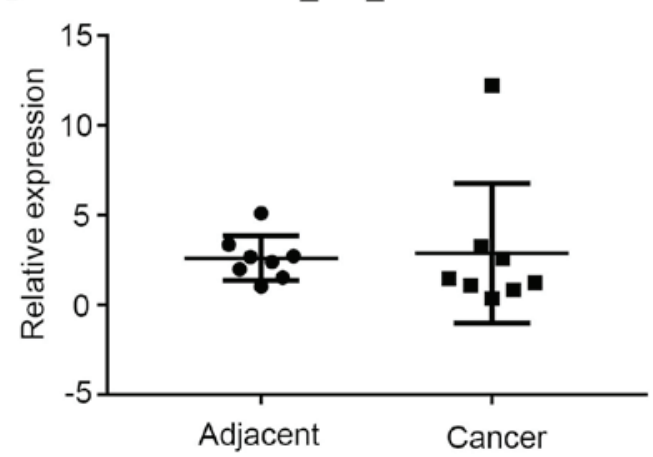

Figure 6. Characterization of hsa_circ_0067531 and hsa_circ_0057096 in HCC tissues. (A) Head-to-tail splicing was assayed by a qPCR reaction, with divergent primers and (B) Sanger sequencing. (C) hsa_circ_0067531 and (D) hsa_circ_0057096 expressions were examined in eight pairs of the HCC tissues and in the corresponding non-tumor tissues using qPCR. The expression of hsa_circ_0067531 in HCC tissues was significantly decreased compared with adjacent normal tissues $(\mathrm{P}=0.012)$, however there was no significant difference in hsa_circ_0057096 expression between adjacent normal tissues and HCC tissues. ${ }^{*} \mathrm{P}<0.05$ vs. adjacent. HCC, hepatocellular carcinoma; qPCR, quantitative polymerase chain reaction.

Table I. Sequences of two differentiated expression circular RNAs from PDK1 and PIK3CB.

\begin{tabular}{lll}
\hline circBASE ID & Gene & Sequence (5'-3') \\
\hline hsa_circ_0057096 & PDK1 & CTTTACAGATACTGTGATACGGATCAGAAACCGACACAATGATGTCATTCCCACA \\
& & ATGGCCCAGGGTGTGATTGAATACAAGGAGAGCTTTGGGGTGGATCCTGTCACC \\
& AGCCAGAATGTTCAGTACTTTTGGATCGATTCTACATGAGTCGCATTTCAATTAG \\
& AATGTTACTCAATCAGCACTCTTTATTGTTTGGTGGAAAGGCAAAGGAAGTCCA \\
& TCTCATCGAAAACACATTGGAAGCATAAATCCAAACTGCAAT GTACTTGAAGTTA \\
& TTAAAG
\end{tabular}

hsa_circ_0067531 PIK3CB AGTCGAGGTGGAAAAAAGTTTCTTCCTGTATTGAAAGAAATCTTGGACAGGGAT CCСTTGTCTCAACTGTGTGAAAATGAAATGGATCTTATTTGGACTTTGCGACAAG ACTGCCGAGAGATTTTCCCACAATCACTGCCAAAATTACTGCTGTCAATCAAGTG GAATAAACTTGAGGATGTTGCTCAGCTTCAGGCGCTGCTTCAGATTTGGCCTAAA CTGCCCCCCCGGGAGGCCCTAGAGCTTCTGGATTTCAACTATCCAGACCAGTACG TTCGAGAATATGCTGTAGGCTGCCTGCGACAGATGAG

PDK1, pyruvate dehydrogenase kinase; PIK3CB, phosphatidylinositol-4,5-bisphosphate 3-kinase catalytic subunit $\beta$.

was performed which provided more detailed information regarding the pathways in which the target genes were involved. It was revealed that the key signaling pathways were the metabolic pathway, pathways in cancer, and the phosphoinositide 3-kinase (P13K)-RAC- $\alpha$ serine/threonine-protein kinase (AKT) signaling pathway (Fig. 5B).

Characterizationofhsa_circ_0067531andhsa_circ_0057096 in $H C C$. To verify the microarray data, two circRNAs with differential expression were selected from the
P13K-AKT signaling pathway; hsa_circ_0067531 and hsa_ circ_0057096. The sequences of these two circRNAs from pyruvate dehydrogenase kinase (PDK) 1 and phosphatidylinositol-4,5-bisphosphate 3-kinase catalytic subunit $\beta$ (PIK3CB) are listed in Table I. Divergent primers amplify circRNAs in cDNA, however not genomic DNA (gDNA) (Fig. 6A). The Sanger sequencing verified head-to-tail splicing (Fig. 6B). The present study validated the expression levels via qPCR in eight pairs of HCC and adjacent normal tissues. It was demonstrated that the expression of hsa_circ_0067531 was 
significantly decreased in the HCC tissues, compared with the normal tissues $(\mathrm{P}=0.012)$ (Fig. 6C), however there was no significant difference in hsa_circ_0057096 expression between adjacent normal tissues and HCC tissues (Fig. 6D). Therefore, it was hypothesized that hsa_circ_0067531 may be a promising target for HCC diagnosis and therapy in the future.

\section{Discussion}

circRNAs have previously emerged as novel and crucial layers of gene regulation and have demonstrated the potential to be ideal biomarkers in the diagnosis of various cancers $(25,26)$. Certain circRNAs have already been demonstrated to exhibit important roles in cancer, including hsa_circ_002059 in gastric cancer, Cdrlas in hepatocellular carcinoma, and cir-ITCH in esophageal squamous cancer $(23,27,28)$. Previous studies have revealed that circRNAs may be involved in the progression of cancer (20). Bachmayr-Heyda, et al (29) identified that circRNAs are significantly downregulated in colorectal cancer (CRC) tissues compared with the normal colon mucosa. Li et al (28) demonstrated that hsa_circ_002059 expression is downregulated in gastric cancer and may be a potential biomarker for its diagnosis (28). Huang et al reported that the cir-ITCH expression is decreased in esophageal squamous cell carcinoma (ESCC) and CRC, and may have an inhibitory effect on ESCC and CRC (30). The mechanisms of circRNAs in $\mathrm{HCC}$ remain to be elucidated.

The primary outcome of the present study was that CD90 promoted cell migration, viability and sphere-forming abilities in HCC, and the secondary outcome was that hsa_circ_0067531 and the PI3K pathway may potentially be involved in the aforementioned process. CD90 is a 25-37 kDa glycophosphatidylinositol-anchored protein that operates as an important regulator of cell-to-cell and cell-to-matrix interactions in cancer (13). CD90 expression has been suggested to be associated with poor HCC prognosis (31-33) and $\mathrm{CD}^{\circ} 0^{+} \mathrm{CSCs}$, however CD90CSCs from HCC cell lines, tumor tissues, or peripheral blood, have not been reported to exhibit tumorigenic and metastatic capabilities $(12,14,15)$. The present study first examined the expression of CD90 in the 4 human HCC cell lines, and demonstrated that CD90 expression was significantly upregulated in the HCC cell line SK-Hep-1. In addition, the data demonstrated that $\mathrm{CD} 90^{+}$cells isolated from the SK-Hep-1 cell line exhibited increased viability, migration and invasive capabilities compared with CD90- cells. The results were consistent with previous research which suggests that $\mathrm{CD}^{+} 0^{+}$cells isolated from HCC tumor tissues have the capacity to generate tumor nodules in immunodeficient mice, whereas CD90- cells do not (12,34,35).

circRNAs have previously been demonstrated to exhibit important roles in HCC cancer, including Circular RNA MTO1, Hsa_circ_0001649, and circZKSCAN1 (36-38). In the present study, 274 differentially expressed circRNAs (including upregulated and downregulated genes) were identified in $\mathrm{CD} 90^{+} \mathrm{HCC}$ cells compared with $\mathrm{CD}^{-} 0^{-} \mathrm{HCC}$ cells via a high throughput microarray assay. Furthermore, KEGG pathway analyses was performed in order to further understand the biological functions of the differentially expressed
circRNA. The results demonstrated that the key signaling pathways were the metabolic pathway, pathways in cancer, and the PI3K-AKT pathway. Of the significantly enriched pathways in the KEGG pathway analysis, PI3K signaling was of interest as it exhibits an important role in HCC cell cycle progression and viability $(39,40)$. Deregulation of the PI3K/AKT signaling pathway has previously been identified in HCC (41), and Rab31, a member of the Ras superfamily, has been reported to have a role in tumor development and progression (42). The PI3K/AKT pathway was revealed to be involved in the Rab31 promotion of HCC progression (43). Previous research has revealed that inhibiting the activation of the PI3K pathway blocks the carcinogenesis and progression of HCC cells $(44,45)$. The present study therefore selected two differentially expressed circRNAs, shsa_circ_0067531 and hsa_circ_0057096, from PDK1 and PIK3CB, respectively. It was demonstrated that hsa_circ_0067531 was markedly downregulated in HCC tissues compared with adjacent normal tissues.

In conclusion, the results of the present study suggested that CD90 may be used as a potential biomarker for HCC. It was revealed that CD90 promoted cell migration, viability and sphere-forming abilities of HCC. In addition, the expression of hsa_circ_0067531 was significantly decreased in HCC compared with adjacent normal tissues, and this suggested that hsa_circ_0067531 may be involved in the development of HCC, at least in part, through the PI3K pathway. However, the present study does not functionally verify how hsa_circ_0067531 affects HCC cell biological functions, and did not verify the involvement of the metabolic and cancer pathways in tumorigenesis of $\mathrm{CD}^{+} 0^{+} \mathrm{HCC}$. The authors aim to investigate the mechanism underlying the modulation of hsa_circ_0067531 on HCC stem cell properties, and investigate the functional roles of the metabolic and cancer pathways in HCC development in future studies.

\section{Acknowledgements}

The present study was supported by the Major Projects on Collaborative Innovation of Industry, Guangzhou (grant no. 201508020076). The authors gratefully acknowledge the assistance of the Department of Hepatopancreatobiliary Surgery for their help in collecting medical records. In addition, the authors would like to thank all the participants of the study, without whom the study would not have been possible.

\section{References}

1. Asia-Pacific Working Party on Prevention of Hepatocellular Carcinoma: Prevention of hepatocellular carcinoma in the Asia-Pacific region: Consensus statements. J Gastroenterol Hepatol 25: 657-663, 2010.

2. Li Z, Zhang C, Lou C, Yan F, Mao Y, Hong X and Zhang Y: Comparison of percutaneous cryosurgery and surgical resection for the treatment of small hepatocellular carcinoma. Oncol Lett 6: 239-245, 2013.

3. Torre LA, Bray F, Siegel RL, Ferlay J, Lortet-Tieulent J and Jemal A: Global cancer statistics, 2012. CA Cancer J Clin 65: 87-108, 2015.

4. Bruix J, Reig M and Sherman M: Evidence-based diagnosis, staging, and treatment of patients with hepatocellular carcinoma. Gastroenterology 150: 835-853, 2016.

5. Kinoshita A, Koike K and Nishino H: Clinical features and prognosis of elderly patients with hepatocellular carcinoma not indicated for surgical resection. Geriatr Gerontol Int 17: 189-201, 2017. 
6. Al-Hajj M, Wicha MS, Benito-Hernandez A, Morrison SJ and Clarke MF: Prospective identification of tumorigenic breast cancer cells. Proc Natl Acad Sci USA 100: 3983-3988, 2003.

7. Collins AT, Berry PA, Hyde C, Stower MJ and Maitland NJ: Prospective identification of tumorigenic prostate cancer stem cells. Cancer Res 65: 10946-10951, 2005.

8. Li C, Heidt DG, Dalerba P, Burant CF, Zhang L, Adsay V, Wicha M, Clarke MF and Simeone DM: Identification of pancreatic cancer stem cells. Cancer Res 67: 1030-1037, 2007.

9. O'Brien CA, Pollett A, Gallinger S and Dick JE: A human colon cancer cell capable of initiating tumour growth in immunodeficient mice. Nature 445: 106-110, 2007.

10. Ricci-Vitiani L, Lombardi DG, Pilozzi E, Biffoni M, Todaro M Peschle C and De Maria R: Identification and expansion of human colon-cancer-initiating cells. Nature 445: 111-115, 2007.

11. Singh SK, Hawkins C, Clarke ID, Squire JA, Bayani J, Hide T, Henkelman RM, Cusimano MD and Dirks PB: Identification of human brain tumour initiating cells. Nature 432: 396-401, 2004.

12. Yang ZF, Ho DW, Ng MN, Lau CK, Yu WC, Ngai P, Chu PW, Lam CT, Poon RT and Fan ST: Significance of CD90 cancer stem cells in human liver cancer. Cancer Cell 13: 153-166, 2008.

13. Rege TA and Hagood JS: Thy-1 as a regulator of cell-cell and cell-matrix interactions in axon regeneration, apoptosis, adhesion, migration, cancer, and fibrosis. FASEB J 20: 1045-1054, 2006.

14. Yamashita T, Honda M, Nakamoto Y, Baba M, Nio K, Hara Y, Zeng SS, Hayashi T, Kondo M, Takatori H, et al: Discrete nature of $\mathrm{EpCAM}^{+}$and $\mathrm{CD} 90^{+}$cancer stem cells in human hepatocellular carcinoma. Hepatology 57: 1484-1497, 2013.

15. Yang ZF, Ngai P, Ho DW, Yu WC, Ng MN, Lau CK, Li ML, Tam KH, Lam CT, Poon RT and Fan ST: Identification of local and circulating cancer stem cells in human liver cancer. Hepatology 47: 919-928, 2008.

16. Sukowati CH, Anfuso B, Torre G, Francalanci P, Crocè LS and Tiribelli C: The expression of CD90/Thy-1 in hepatocellular carcinoma: An in vivo and in vitro study. PLoS One 8: e76830, 2013.

17. Chen WC, Hsu HP, Li CY, Yang YJ, Hung YH, Cho CY, Wang CY, Weng TY and Lai MD: Cancer stem cell marker CD90 inhibits ovarian cancer formation via $\beta 3$ integrin. Int J Oncol 49: 1881-1889, 2016.

18. Wang F, Nazarali AJ and Ji S: Circular RNAs as potential biomarkers for cancer diagnosis and therapy. Am J Cancer Res 6 : 1167-1176, 2016.

19. Li J, Yang J, Zhou P, Le Y, Zhou C, Wang S, Xu D, Lin HK and Gong Z: Circular RNAs in cancer: Novel insights into origins, properties, functions and implications. Am J Cancer Res 5: 472-480, 2015

20. Qu S, Yang X, Li X, Wang J, Gao Y, Shang R, Sun W, Dou K and Li H: Circular RNA: A new star of noncoding RNAs. Cancer Lett 365: 141-148, 2015.

21. Kulcheski FR, Christoff AP and Margis R: Circular RNAs are miRNA sponges and can be used as a new class of biomarker. J Biotechnol 238: 42-51, 2016.

22. Fu L, Yao T, Chen Q, Mo X, Hu Y and Guo J: Screening differential circular RNA expression profiles reveals hsa_circ_0004018 is associated with hepatocellular carcinoma. Oncotarget 8: 58405-58416, 2017.

23. Yu L, Gong X, Sun L, Zhou Q, Lu B and Zhu L: The Circular RNA Cdrlas Act as an Oncogene in Hepatocellular carcinoma through targeting miR-7 expression. PLoS One 11: e0158347, 2016.

24. Schmittgen TD: Real-time quantitative PCR. Methods 25: 383-385, 2001.

25. Chen LL and Yang L: Regulation of circRNA biogenesis. RNA Biol 12: 381-388, 2015.

26. Hansen TB, Jensen TI, Clausen BH, Bramsen JB, Finsen B, Damgaard CK and Kjems J: Natural RNA circles function as efficient microRNA sponges. Nature 495: 384-388, 2013.

27. Li F, Zhang L, Li W, Deng J, Zheng J, An M, Lu J and Zhou Y: Circular RNA ITCH has inhibitory effect on ESCC by suppressing the $\mathrm{Wnt} / \beta$-catenin pathway. Oncotarget 6 : 6001-6013, 2015 .

28. Li P, Chen S, Chen H, Mo X, Li T, Shao Y, Xiao B and Guo J: Using circular RNA as a novel type of biomarker in the screening of gastric cancer. Clin Chim Acta 444: 132-136, 2015.
29. Bachmayr-Heyda A, Reiner AT, Auer K, Sukhbaatar N, Aust S, Bachleitner-Hofmann T, Mesteri I, Grunt TW, Zeillinger R and Pils D: Correlation of circular RNA abundance with viability-exemplified with colorectal and ovarian cancer, idiopathic lung fibrosis, and normal human tissues. Sci Rep 5: 8057, 2015.

30. Huang G, Zhu H, Shi Y, Wu W, Cai H and Chen X: cir-ITCH plays an inhibitory role in colorectal cancer by regulating the Wnt/ $\beta$-catenin pathway. PLoS One 10: e0131225, 2015.

31. Lingala S, Cui YY, Chen X, Ruebner BH, Qian XF, Zern MA and $\mathrm{Wu} \mathrm{J}$ : Immunohistochemical staining of cancer stem cell markers in hepatocellular carcinoma. Exp Mol Pathol 89: 27-35, 2010.

32. Lu JW, Chang JG, Yeh KT, Chen RM, Tsai JJ and Hu RM: Overexpression of Thy1/CD90 in human hepatocellular carcinoma is associated with HBV infection and poor prognosis. Acta Histochem 113: 833-838, 2011.

33. Yu XH, Xu LB, Liu C, Zhang R and Wang J: Clinicopathological characteristics of 20 cases of hepatocellular carcinoma with bile duct tumor thrombi Dig Dis Sci 56: 252-259, 2011.

34. Ho DW, Yang ZF, Yi K, Lam CT, Ng MN, Yu WC, Lau J, Wan T, Wang X, Yan Z, et al: Gene expression profiling of liver cancer stem cells by RNA-sequencing. PLoS One 7: e37159, 2012.

35. Yang R, An LY, Miao QF, Li FM, Han Y, Wang HX, Liu DP, Chen $R$ and Tang SQ: Effective elimination of liver cancer stem-like cells by CD90 antibody targeted thermosensitive magnetoliposomes. Oncotarget 7: 35894-35916, 2016.

36. Han D, Li J, Wang H, Su X, Hou J, Gu Y, Qian C, Lin Y, Liu X, Huang M, et al: Circular RNA circMTO1 acts as the sponge of miR-9 to suppress hepatocellular carcinoma progression. Hepatology 66: 1151-1164, 2017.

37. Qin M, Liu G, Huo X, Tao X, Sun X, Ge Z, Yang J, Fan J, Liu L and Qin W: Hsa circ 0001649: A circular RNA and potential novel biomarker for hepatocellular carcinoma. Cancer Biomark 16: 161-169, 2016.

38. Yao Z, Luo J, Hu K, Lin J, Huang H, Wang Q, Zhang P, Xiong Z, He C, Huang Z, et al: ZKSCAN1 gene and its related circular RNA (circZKSCAN1) both inhibit hepatocellular carcinoma cell growth, migration, and invasion but through different signaling pathways. Mol Oncol 11: 422-437, 2017.

39. Fulda S: Modulation of mitochondrial apoptosis by PI3K inhibitors. Mitochondrion 13: 195-198, 2013.

40. Fulda S: Synthetic lethality by co-targeting mitochondrial apoptosis and PI3K/Akt/mTOR signaling. Mitochondrion 19: 85-87, 2014.

41. Gedaly R, Angulo P, Hundley J, Daily MF, Chen C and Evers BM: PKI-587 and sorafenib targeting PI3K/AKT/mTOR and Ras/Raf/MAPK pathways synergistically inhibit HCC cell viability. J Surg Res 176: 542-548, 2012.

42. Pan Y, Zhang Y, Chen L, Liu Y, Feng Y and Yan J: The critical role of Rab31 in cell viability and apoptosis in cancer progression. Mol Neurobiol 53: 4431-4437, 2016.

43. Sui Y, Zheng $X$ and Zhao D: Rab31 promoted hepatocellular carcinoma (HCC) progression via inhibition of cell apoptosis induced by $\mathrm{PI} 3 \mathrm{~K} / \mathrm{AK} \mathrm{T} / \mathrm{Bcl}-2 / \mathrm{BAX}$ pathway. Tumor Biol 36: 8661-8670, 2015

44. Liang M, Liu J, Ji H, Chen M, Zhao Y, Li S, Zhang X and Li J: A Aconitum coreanum polysaccharide fraction induces apoptosis of hepatocellular carcinoma (HCC) cells via pituitary tumor transforming gene 1 (PTTG1)-mediated suppression of the P13K/Akt and activation of p38 MAPK signaling pathway and displays antitumor activity in vivo. Tumour Biol 36: 7085-7091, 2015.

45. Zheng J, Li C, Wu X, Liu M, Sun X, Yang Y, Hao M, Sheng S, Sun Y,Zhang H, et al: Astrocyte elevated gene-1 (AEG-1) shRNA sensitizes Huaier polysaccharide (HP)-induced anti-metastatic potency via inactivating downstream P13K/Akt pathway as well as augmenting cell-mediated immune response. Tumour Biol 35: 4219-4224, 2014

This work is licensed under a Creative Commons Attribution-NonCommercial-NoDerivatives 4.0 International (CC BY-NC-ND 4.0) License. 